\title{
Ventricular Arrythmias in Cardiac Amyloidosis: It is Possible to Prevent Sudden Death?
}

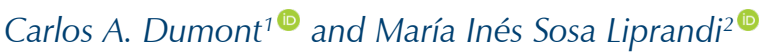 \\ On behalf of the Council of Myocardiopathies of the Inter-American Society of Cardiology \\ Heart Failure Unit, Hospital Privado de Rosario, ${ }^{1}$ Rosario - Argentina \\ Cardiovascular Research and Heart Failure Unit, ${ }^{2}$ Sanatorio Güemes, Buenos Aires - Argentina
}

\begin{abstract}
Cardiac amyloidosis (CA) is an infiltrative cardiomyopathy that occurs secondary to deposition of misfolded protein in the myocardium. The two most common subtypes are amyloid light chain amyloidosis and amyloid transthyretin amyloidosis. CA frequently results in congestive cardiac failure and arrhythmias, due to a disruption in cardiac substrate with subsequent electromechanical remodeling. Disease progression is usually demonstrated by development of progressive pump failure, which may be seen with a high arrhythmic burden, usually portending a poor prognosis. Arrhythmias are common, and many commonly used pharmacological therapies may be poorly tolerated and lead to clinical decompensation in this population, adding complexity to the co-management of these conditions. Studies are required to assess the risks and benefits of catheter ablation for ventricular tachycardia, with no current data showing a mortality benefit. The role of implantable cardioverter-defibrillator therapy is controversial, with benefits seen predominantly in early phases of the disease process. High-quality evidence and guideline recommendations are limited with regard to the management of arrhythmias. Providers are often left to clinical experience and expert consensus to aid in decision-making. This review summarizes both historical and contemporary data and describes evidence-based strategies for managing ventricular arrhythmias and their sequelae in patients with CA.
\end{abstract}

\section{Introduction}

Amyloidosis is a systemic disease characterized by the extracellular deposition of pathological insoluble fibrillar protein, known as amyloid, within various organs (mainly the heart and kidneys). ${ }^{1}$ The leading cause of morbidity and mortality in these patients is from development of restrictive cardiomyopathy with progressive congestive cardiac failure

\section{Keywords}

Amyloidosis; Tachycardia, Ventricular; Death, Sudden, Cardiac; Defibrillators, Implantable.

\author{
Mailing Address: Carlos A. Dumont • \\ Hospital Privado de Rosario \\ Pte. Roca 2440.CP 2000, Rosario, Santa Fé - Argentina \\ Email: carlosdumont8@gmail.com \\ Manuscript received October 12, 2021, revised manuscript November 01, \\ 2021, accepted November 01, 2021.
}

DOI: https://doi.org/10.36660/abchf.20210035
(CCF). ${ }^{2}$ Approximately $95 \%$ of cases of cardiac amyloidosis (CA) are misfolded transthyretin (ATTR) or immunoglobulin light-chain (AL) types. ${ }^{3}$ The ATTR group is characterized by misfolding of transthyretin, a protein made mainly in the liver. Both wild-type ATTR cardiomyopathy (wtATTR-CM) and hereditary ATTR cardiomyopathy (hATTR-CM) have been described. The prevalence of wtATTR-CM is estimated to be between $5.5 \%$ and $16.0 \%$ of people $>80$ years of age. Recent trends have revealed a significant increase in the number of new diagnoses of ATTR-CM over the past 10 years, suggesting that estimates of disease burden underestimate the true prevalence amyloid cardiomyopathy. ${ }^{3} \mathrm{AL}$ amyloidosis is a clonal plasma cell proliferative disorder characterized by production of abnormal protein fibrils in the bone marrow. The estimated prevalence of $\mathrm{AL}$ amyloidosis in the United States ranges from 15.5 to 40.5 cases per million. ${ }^{1-3}$

CA should be suspected particularly in patients with heart failure with preserved left ventricular ejection fraction presenting "red flag" signs such as (1) either symmetrical or asymmetrical unexplained left and right ventricular hypertrophy with concomitant diastolic dysfunction and reduced left ventricular global longitudinal strain (LV GLS) with an "apical sparing" pattern in echocardiography, (2) discrepancy between the left ventricular (LV) wall thickness and QRS voltage and the presence of pseudoinfarct pattern in electrocardiography, especially if associated with increased levels of $\mathrm{N}$-terminal pro B-type natriuretic peptide (NT-proBNP). ${ }^{4}$

Although novel treatment vastly improved survival in $\mathrm{AL}$ and ATTR CA, cardiovascular events account for more than two thirds of fatal casualties in both groups. ${ }^{5}$ Moreover, sudden cardiac death (SCD) accounts for up to $50 \%$ of all cardiac deaths. ${ }^{6}$ Electromechanical dissociation is thought to be the most common cause of SCD in patients with CA; however, ventricular arrhythmias (VA) and conduction abnormalities are also common. ${ }^{7}$ To date, little is known about risk factors for ventricular tachyarrhythmias as a cause of SCD in patients with amyloidosis and cardiac involvement. Therefore, identification of patients with CA who may be eligible for implantable cardioverter-defibrillator (ICD) is challenging.

While both forms of amyloid subtypes have a predilection to the development of amyloid cardiomyopathy, the underlying electrical burden and arrhythmogenic substrate vary between them, with distinct clinical implications. ${ }^{8}$ The resultant disruption of the cardiac conduction system in patients with CA predisposes to arrhythmias, with the most frequent identified as atrial fibrillation (45\% to $65 \%$ ) and, less frequently, atrioventricular conduction delays (3.5\%) and ventricular tachyarrhythmias (ventricular tachycardia 
$9.9 \%$ and ventricular fibrillation $0.7 \%$, respectively). ${ }^{9,10}$ WATTR appears to carry the highest risk of atrial fibrillation, and commonly used cardiovascular therapeutics such as beta-blockers, calcium-channel blockers, and digoxin may be poorly tolerated and lead to clinical decompensation in $\mathrm{CA}$, adding further complexity to the co-management of these conditions. The literature currently states that patients with AL CA have a higher propensity to develop VA. ${ }^{11}$ The historically reported survival of $<12$ months in patients with cardiac AL has represented a contraindication to ICD. However, recently, the evolution of treatment options has improved overall survival, opening new perspectives in terms of cardiac protection and calling for further understanding of the arrhythmic profile and SCD prevention in patients with AL.

\section{Epidemiology of Ventricular Arrhythmias}

The prevalence of nonsustained ventricular tachycardia (NSVT) in AL amyloidosis ranges from $5 \%$ to $27 \%$ with routine monitoring, ${ }^{11-13}$ and $100 \%$ during the stem cell transplant period. ${ }^{14}$ The prevalence of ventricular tachycardia (VT) in the ATTR population is less frequently reported, but small studies suggest a prevalence of approximately $17 \% .{ }^{15}$ Additionally, as $\mathrm{AL}$ amyloidosis is more likely to cause end-stage CCF when compared to ATTR, there is a higher preponderance to development of VA. The clinical significance of VA is varied, with some studies suggesting a prognostic role ${ }^{11-14}$ and other studies reporting no such correlation. ${ }^{16}$

\section{Pathophysiology of Ventricular Arrhythmias}

There are a variety of mechanisms that form the fundamental pathogenesis for VA in CA. Firstly, amyloid fibril deposition with a resultant inflammatory response and oxidative stress leads to a separation of myocytes resulting in remodeling and LV fibrosis, which progressively develops arrhythmogenic potential. ${ }^{1,2-4}$ Secondly, amyloid fibril deposition in the conduction system can potentiate arrhythmias. ${ }^{17}$ Thirdly, micro- and macrovascular myocardial ischemia from amyloid deposition can be demonstrated by regional myocardial dysfunction, as assessed by cardiac magnetic resonance (CMR). ${ }^{18}$ The cause and mechanisms of VA in CA, however, are poorly understood and are likely to be multifactorial.

\section{Prognosis}

Cardiac involvement is the determinant of prognosis in amyloidosis. Risk of death in patients with $\mathrm{AL}$ amyloidosis can be stratified using the revised Mayo staging models, including cardiac biomarkers: cardiac troponin $\mathrm{T}(\mathrm{cTnT} \geq 0.025 \mathrm{ng} / \mathrm{mL}$ ), NT-proBNP $(\geq 1,800 \mathrm{pg} / \mathrm{mL})$ and serum immunoglobulin free light chain difference $\geq 18 \mathrm{mg} / \mathrm{dL} .{ }^{19}$ Similar to $\mathrm{AL}$ amyloidosis, a staging system including markers of increased myocardial stress, such as NT-proBNP and high sensitivity cTnT, has been proposed for ATTR amyloidosis. Patients with both cTnT > $0.05 \mathrm{ng} / \mathrm{mL}$ and NT-proBNP $>3,000 \mathrm{pg} / \mathrm{mL}$ had the worst prognosis, with median survival of only 20 months. ${ }^{20}$ Additional to elevated cardiac biomarkers, renal dysfunction was also identified as a significant risk factor of worse prognosis. In the recently proposed prognostic system for staging ATTR amyloidosis, patients with decreased estimated glomerular filtation rate $<45 \mathrm{~mL} /$ $\mathrm{min} / 1.73 \mathrm{~m}^{2}$ and NT- proBNP $>3,000 \mathrm{pg} / \mathrm{mL}$ had significantly worse survival compared to those not meeting these cut-off values. ${ }^{21}$ However, the mentioned staging models only predict overall mortality. There is no significant correlation between cardiac biomarkers levels and the risk of VA. ${ }^{22}$

It is crucial to understand the pathophysiology of VA in CA in order to predict the risk of death. Amyloid in the extracellular spaces distorts the myocardial cells, and it can also infiltrate cardiac conduction system and coronary arteries. Besides infiltration, amyloidogenic light chains in $\mathrm{AL}$ amyloidosis may directly impair cardiomyocyte function through an increase in cellular oxidant stress. It appears that myocardial scarring and fibrosis that are typical of chronic ischemic or non-ischemic cardiomyopathies are less common in CA. It is well known that patients with structural heart disease have a lower threshold in developing arrhythmias, and this may be the case for patients with CA, specifically the AL subtype. ${ }^{23}$ Several echocardiographic correlates of structural heart disease are known to result in VT, for example evidence of elevated LV systolic diameter, impaired left ventricular ejection fraction (LVEF < 30\%), widened QRS (> $125 \mathrm{msec}$ ), age > 65 years, prior history of CCF, and active diuretic therapy, when compared to patients without VT. ${ }^{23,24}$ These specific clinical and echocardiographic findings are frequently seen in patients with CA, and, therefore, these high-risk populations should undergo frequent monitoring. ${ }^{24}$ Similarly, a study by Falk et al. has shown that, in patients with CA who present with high-grade VA, there is more likely to be a history of CCF and an abnormal echocardiogram, when compared to patients without arrhythmias. ${ }^{25}$ These findings highlight the relationship between structural heart disease, heart failure, and VT. Therefore, structural assessment in these patients may provide prognostic value and allow for more appropriate medical therapy. In some patients, the qualification for primary ICD implantation is based on the standard LV systolic dysfunction with LVEF $\leq 35 \%$. However, in the majority of patients with $\mathrm{CA}$, the decline of LV systolic function is a late manifestation; therefore, other echocardiographic parameters are needed to assess LV function (even in those with preserved LVEF). Harmon et al. showed that VA was more common in patients with reduced two-dimensional LV GLS ( $\geq-15 \%$ ) assessed by speckle-tracking echocardiography. ${ }^{26}$

CMR not only plays an important role in the diagnosis of CA; it also provides important prognostic information. In amyloidosis, CMR enables myocardial tissue characterization by means of T1- and T2-weighted imaging sequences, T1 mapping (pre- and post-contrast), late gadolinium enhancement (LGE), and extracellular volume (ECV) imaging. Global subendocardial or transmural pattern of LGE and, to a lesser degree, focal patchy LGE are all features of CA. The extent of LGE may also serve as a surrogate of arrhythmogenic substrate for the occurrence of VA. ${ }^{27}$ Two-year survival in patients with CA without LGE was 92\%, whereas it was significantly lower in those who showed subendocardial or transmural LGE (81\% and $45 \%$, respectively). ${ }^{28}$ Both in $\mathrm{AL}$ and ATTR CA, the presence of transmural LGE has been shown to be an independent predictor of worse survival. ${ }^{28}$ However, the limitation of LGE is that it is difficult to quantify, making it 
difficult to track changes in CA, for example, due to treatment. Post-contrast T1 mapping following gadolinium administration enables estimation of ECV. ECV values are significantly elevated in CA, and ECV is a robust prognostic marker in CA. Moreover, the assessment of ECV as well as native T1 values makes it possible to track the disease and response to therapy over time. Additionally, T2 mapping provides data on T2 relaxation times which represent myocardial edema and active inflammation and are potentially linked with arrhythmogenic potential. In a recent study, ${ }^{29}$ the presence of myocardial edema was shown in CA, as indicated by increased T2 relaxation times in patients with amyloidosis compared to control subjects, as well as in untreated $\mathrm{AL}$ amyloidosis compared with treated AL and ATTR amyloidosis. In this study, T2 was a prognostic predictor in AL amyloidosis, which may suggest mechanisms additional to amyloid infiltration contributing to mortality in this disease. ${ }^{23,30}$

There have also been studies assessing the relationship between cardiac monitoring findings and VT in CA. In a recent study of 239 patients with $\mathrm{AL} \mathrm{CA}$, while the presence of NSVT conferred worse survival prior to stem cell transplant, cardiac monitoring only detected its presence in approximately $25 \%$ of patients. ${ }^{31}$ In a prospective series of 20 patients with syncope in the context of severe CA by Sayed et al., only 1 episode of VT was documented, with a total of 13 deaths. The remaining arrhythmias detected were either atrial arrhythmias or atrioventricular conduction block. ${ }^{12}$ NSVT is a common finding among patients with $\mathrm{CA}$, and its role in predicting SCD in this population is debated, as it appears to have little discriminative value to identify those who die from VA. ${ }^{26,32}$ In the meta-analysis by Halawa et al., despite high prevalence of NSVT (in $51 \%$ of patients with CA), only $18 \%$ received appropriate ICD therapy. ${ }^{33}$ Nevertheless, NSVT in the early stage of $\mathrm{AL}$ amyloidosis can be considered an indication for ICD implantation in primary prevention (class IIb). ${ }^{34}$

To better understand the underlying pathophysiology, Orini et al. combined the assessment of the electrophysiological and structural ventricular substrate from 21 patients with CA (11 AL and 10 ATTR). ${ }^{35}$ The authors used a special electrocardiographic system with 256 electrodes for non-invasive epicardial mapping of ventricular potentials and CMR imaging. When compared with healthy volunteers, patients with CA had significantly lower epicardial signal amplitude, slower heterogeneous intraventricular conduction, and prolonged and more spatially dispersed repolarization. Moreover, epicardial signal fractionation and average repolarization time increased with ECV calculated in CMR. A strong inverse correlation was found between epicardial signal amplitude and native T1 in CMR. Both epicardial conduction and repolarization abnormalities were more notable in patients with $\mathrm{AL}$ amyloidosis compared with ATTR. Spatial conduction-repolarization heterogeneity is thought to be a marker of increased propensity to VA and sudden arrhythmic death in patients with heart failure and may contribute to higher mortality in $\mathrm{AL}$ amyloidosis. ${ }^{36}$ This study also suggests a link between conductionrepolarization delay and increased extracellular deposition.

Invasive electrophysiological study is infrequently performed in patients with CA. Reisinger at al. demonstrated a prolongation of the His-ventricular (HV) interval > $55 \mathrm{~ms}$ in the majority of the examined population ( 23 of 25 patients with biopsy-confirmed AL amyloidosis), which indicated disease of the distal His-Purkinje system. ${ }^{6}$ Markedly prolonged HV interval ( $\geq 80 \mathrm{~ms}$ ) was the only independent predictor for SCD in the multivariate analysis. The authors concluded that prolongation of the HV interval not only indicates a risk of complete atrioventricular block due to conduction system infiltration with amyloid fibrils and bradyarrhythmia as a potential cause of death; it may also indicate severe myocardial infiltration and serve as a marker of the propensity for lethal VA or acute electromechanical dissociation. Interestingly, in this study, monomorphic VT was induced in only 4 patients during programmed ventricular stimulation, and, similarly to other non-ischemic cardiomyopathies, VT non-inducibility showed little prognostic value.

Amyloid infiltration is more severe in ATTR when compared to $\mathrm{AL}$ amyloidosis, which ultimately results in greater $\mathrm{LV}$ mass and higher rates of $\mathrm{CCF}^{2}$ In spite of these phenotypic differences, AL amyloidosis confers worse prognosis with high rates of SCD (approximately 33\% of patients) in the first 3 months of diagnosis. ${ }^{12}$ This may be secondary to higher spatial heterogenous conduction and repolarization, which is a marker of arrhythmogenesis and overall worse prognosis, as shown by Orini et al. ${ }^{35}$

\section{Management}

The management of VA in CA requires a different approach to standard VA guidelines in the context of heart failure or conventional cardiomyopathies. The treatment of VT can be subdivided into cardiac antiarrhythmic medications, procedural-based therapy, and surgical modalities. Patients presenting with VT frequently undergo extensive investigation, including CMR imaging to evaluate myocardial LGE or scarring, as it provides prognostic information and may identify a site of origin of VT that can then be effectively targeted by catheter ablation. ${ }^{37}$ This work-up may be beneficial in patients with CA presenting with VA. Conventional pharmacotherapies that are used in treatment of VT include oral antiarrhythmic agents and beta-blockade. Beta-blockers, while used widely in the suppression of VT with beta-adrenergic antagonist activity, may be detrimental with a resultant loss in cardiac output, in patients with CA. ${ }^{9}$ Amiodarone, another commonly used and effective therapy for VT, may result in complications when treating patients with CA, namely complete heart block when compared to patients without CA, as shown in a study $(43.8 \%$ versus $30.0 \%, P<0.0001) .{ }^{38}$ These findings indicate poorer overall outcomes with conventional pharmacological therapy for VT, when used in patients with CA.

Chemotherapy for $\mathrm{AL}$ amyloidosis is mainly based on regimens used for the treatment of myeloma and not specifically approved for AL cardiac involvement. Most of these drugs have established cardiotoxic potential, with increased risk of heart failure and arrhythmic events. A study by Le Bras et al. demonstrated that dexamethasone as an induction agent for treatment of patients with AL CA may potentiate fluid retention and promote arrhythmias, hence resulting in an arrhythmogenic ventricular substrate. ${ }^{39}$ Similarly, high dose chemotherapy, specifically cyclophosphamide and bortezomib, 
in $\mathrm{AL}$ amyloidosis can be cardiotoxic in nature and result in VT due to myocardial dysfunction. ${ }^{40,41}$

While the role of catheter ablation for treatment of atrial arrhythmias is evolving, with the current literature showing potential benefits in terms of recurrence rate and overall survival, there is a lack of data on its use in VA. ${ }^{42,43}$ Other than case reports of successful radiofrequency VT ablation, there have been no large-scale studies assessing the role of VT or ventricular fibrillation ablation in CA. ${ }^{44,45}$ Catheter ablation of VT has been shown to have some utility in patients with other infiltrative cardiomyopathies, such as cardiac sarcoidosis, and it may, therefore, be a viable option for patients with CA. ${ }^{46}$ Further large-scale studies are required to assess the risk and benefits of catheter ablation for VT in CA, with no current data showing a mortality benefit.

The development of refractory heart failure is usually seen with incessant VT and portends a poor prognosis. Epicardial mapping and catheter ablation are also options for management of incessant VT. ${ }^{47}$ The definitive surgical therapy for treatment of end-stage heart failure, which may be seen with recurrent VA, is orthotopic cardiac transplantation, an option in the setting of failed medical management. ${ }^{48}$ Heart transplantation is an effective therapy for end-stage CA with good follow-up survival rates. ${ }^{49}$

The advent of disease modifying therapies for ATTR has revolutionized management protocols. Tafamidis, a transthyretin stabiliser, prevents overall tetramer dissociation and amyloidogenesis; therefore, it has been shown to reduce all-cause mortality and cardiovascular hospitalizations. ${ }^{50}$ Similarly, diflusinal is a non-steroidal drug that also stabilizes the transthyretin tetramer and has been shown to stabilize LV mass, indicating a halt in progression of cardiac amyloidogenesis. ${ }^{51}$ By effectively preventing amyloidogenesis, these therapies may result in reduced incidence of SCD with the potential to mitigate VA in the population with ATTR.

\section{Device Therapy}

Whether there is a selected population of patients with CA at risk of arrhythmic SCD (versus SCD due to electromechanical dissociation) who would benefit from ICD placement is still a matter of debate. ${ }^{52}$ Currently, there is no consensus on the absolute benefit that ICDs can confer patients with CA. While its potential to eliminate fatal VA is supported by select and anecdotal cases, the overall effectiveness of ICDs and their safety remains unclear. ${ }^{52}$ Most decisions made regarding ICD insertions are largely patient-centered and based upon local expertise.

Studies have shown that up to one half of patients with CA die suddenly; however, ICD placement for both primary and secondary prevention in CA has not been strongly supported by expert guidelines. Reasons for this include the following: 1) the most common cause of sudden death has been historically thought to be secondary to electromechanical dissociation resulting in pulseless electrical activity rather than a lethal VA; 2) suggestion by some that CA carries a higher defibrillation threshold that may be refractory to ICD therapy; and 3) a historically poor prognosis and life expectancy in this population. ${ }^{12,16}$
The 2015 European Society of Cardiology (ESC) Guidelines for management of VA and prevention of SCD state that "there are insufficient data to provide recommendations" for the use of ICDs for primary prevention of SCD in patients with CA..$^{53}$ The ESC gives class Ila, level of evidence C, for ICD implantation in secondary prevention, recommending consideration for those with either AL or hATTR CA and "ventricular arrhythmia causing hemodynamic instability who are expected to survive $>$ 1 year with good functional status." The 2017 American Heart Association/American College of Cardiology/Heart Rhythm Society guideline for management of patients with VA and prevention of SCD recommends individualized decision-making for both primary and secondary prevention ICD placement in CA, as data remain too limited to allow formal recommendations. ${ }^{54}$ According to the Heart Rhythm Society consensus statement from 2019, a prophylactic ICD may be considered in patients with NSVT in the course of AL and expected survival more than 1 year. ${ }^{35}$ However, it is only a class IIb recommendation. Both the ESC and American Heart Association guidelines are consistent in recommending against placement of ICD for primary or secondary prevention in patients with heart failure and expected meaningful survival of $<1$ year, including in those with medication-refractory New York Heart Association functional class IV heart failure who are not also candidates for cardiac resynchronization therapy, cardiac transplantation, or LV assist devices.

Retrospective analyses (Table 1) of outcomes in patients with CA implanted with ICD for primary and secondary prevention have shown that almost one quarter of patients received appropriate ICD therapy, and the incidence of inappropriate ICD interventions was low (7\%). However, only one quarter of patients who received appropriate ICD therapy survived the follow-up, and in almost two thirds of patients the ICD probably had no effect on their survival. Kristen et al..$^{55}$ found that only $11 \%$ of 19 patients with AL CA implanted with ICD for primary prevention received appropriate ICD therapies for sustained ventricular tachyarrhythmias. Lin et al. ${ }^{32}$ from the Mayo Clinic found that $28 \%$ of 53 patients received appropriate ICD shocks at 1 year. Patients with AL CA comprised most of those who received appropriate shocks (12 of 15 patients), and those who were shocked more often had received an ICD for secondary prevention. ICD therapy was not associated with improved mortality in follow-up. Varr et al. ${ }^{22}$ from Stanford University found that $26 \%$ of 19 patients received appropriate shocks in follow-up. In this study, not a single patient who received an ICD for primary prevention received appropriate successful ICD therapy. There are multiple limitations to these studies. First, all of the studies included in this review were retrospective with various sample sizes. Second, the reports included patients with a variety of CA etiologies, including AL and ATTR, while each has a different clinical presentation, natural history, prognosis, and treatment. Regarding prognosis, Harmon et al. indicated in multivariate analysis that $\mathrm{AL}$ amyloidosis was an independent predictor of high mortality in patients with $\mathrm{CA} .{ }^{26}$ Third, the majority of ICDs were implanted in primary prevention for patients who had LVEF $<35 \%$. Qualification was mostly based on arbitrarily adopted criteria, which included the presence of different types of VA (such as NSVT or frequent premature ventricular beats) and/or non-postural syncope. Although the detection of NSVTs and reports of syncope should prompt immediate clinical concerns, the predictive value for primary SCD prevention 
is controversial. Unexplained syncope is a common and non-specific symptom in CA population, and it can result from other causes than conduction disturbances or VA, such as orthostatic hypotension, autonomic dysfunction, or the use of diuretics or vasodilating drugs. ${ }^{16}$

There is an increasing awareness that the primary driver of mortality in these patients is electromechanical dissociation, as opposed to VT. ${ }^{56,57}$ Electromechanical dissociation is likely a manifestation of progressive deterioration in heart failure not amenable to ICD therapy. ${ }^{55} \mathrm{CA}$ is often diagnosed late in various stages of amyloidosis, and overall mortality is higher in advanced stages of the disease. It is noteworthy that patients with greater cardiac involvement, manifested as higher NT-proBNP concentration and lower LVEF, may be at higher risk of death from electromechanical dissociation. ${ }^{22}$ Therefore, ICD implantation can be more beneficial in patients with cardiac involvement but with lower NT-proBNP level and preserved LVEF. ${ }^{32}$

It is known that the risk of ventricular tachyarrhythmias and SCD varies with the type of CA and is significantly greater in patients with $\mathrm{AL}$ amyloidosis. ${ }^{52}$ Based on the current state of knowledge, there are no robust guidelines for the decision to implant an ICD for primary prevention in CA. When NSVT and syncope are captured and documented, ICD implantation for prevention of SCD in CA is most likely a reasonable approach. ${ }^{57}$ Specifically, this would be most appropriate for patients with $\mathrm{AL}$ amyloidosis, where ICD implantation is associated with a high rate of appropriate discharges for VA. An algorithm for ICD implantation could be proposed in patients with AL amyloidosis and LVEF > 35\% (Figure 1). ICD should be considered in patients with registered NSVT and in early stages of the disease with less impairment in cardiac function, as indicated by minimally to moderately elevated cardiac biomarkers. As NSVT has been documented to be a poor predictor of SCD in AL amyloidosis, the incidence of syncope, decreased LV GLS in echocardiography, and the presence of transmural LGE in CMR can further improve stratification. Finally, specifically for AL amyloidosis, ICD implantation for primary prevention may also be reasonable for patients awaiting heart transplantation or a mechanical LV assist device, either as destination therapy or bridge-totransplantation. This is reasonable only if perceived survival is meaningful (greater than 1 year), NT-pro BNP is less than $8,500 \mathrm{ng} / \mathrm{L}$ and NYHA functional class is less than IV. ${ }^{58}$

Conduction disease is highly prevalent in CA, and atrioventricular conduction delay involving the His-Purkinje system appears to be more common than pure sinus node disease. ${ }^{6,59} \mathrm{HV}$ interval prolongation with relatively narrow QRS duration may suggest frequent widespread involvement of both right and left bundle branches in patients with CA. Permanent pacemakers are commonly indicated for patients with CA and significant conduction disease, most often in wtATTR. ${ }^{3}$ Donnellan et al. ${ }^{60}$ from the Cleveland Clinic found that routine use of cardiac resynchronization therapy was associated with improvements in mitral regurgitation severity (67\% of patients), New York Heart Association functional class $(67 \%$ of patients), and LVEF (78\% of patients) in patients with ATTR CA and an indication for permanent pacemaker.

\section{Future Directions}

Novel medical therapies for both AL and ATTR CA are leading to increased survival. These management paradigms are aimed at reducing overall arrhythmogenic burden in this population group. ${ }^{61,62}$ As patients with CA are living longer, future studies on arrhythmia management in this population are needed. Questions that need answering include the following: 1)What clinical factors predict lethal VA in this population? 2) What is the appropriate patient

Table 1 - Studies reporting on treatment with implantable cardioverter-defibrillator and survival in patients with cardiac amyloidosis

\begin{tabular}{|c|c|c|c|c|c|c|}
\hline Study/Year Published & $\begin{array}{l}\text { CA } \\
\text { Patients } \\
\text { with ICD } \\
\text { (n) }\end{array}$ & $\begin{array}{c}\mathrm{AL} \\
\text { Amyloidosis }\end{array}$ & $\begin{array}{l}\text { ICD in Primary } \\
\text { Prevention } \\
(\mathrm{n} / \%)\end{array}$ & $\begin{array}{l}\text { Criteria for ICD Implantation in } \\
\text { Primary Prevention }\end{array}$ & $\begin{array}{l}\text { Appropriate } \\
\text { ICD Therapy } \\
(n / \%)\end{array}$ & $\begin{array}{c}\text { Overall } \\
\text { Survival } \\
(n / \%)\end{array}$ \\
\hline Kristen et al. (2008) [55] & 19 & $19 / 19(100 \%)$ & $19(100 \%)$ & Syncope and/or frequent PVBs & $2(11 \%)$ & $1 / 2(50 \%)$ \\
\hline Lin et al. (2013) [32] & 53 & $33 / 53(62 \%)$ & $41(77 \%)$ & LVEF $\leq 35 \%$ or syncope or NSVT & $15(28 \%)$ & $21 / 53(40 \%)$ \\
\hline Varr et al. (2014) [22] & 19 & $15 / 19(79 \%)$ & $15(79 \%)$ & Not specified & $5(26 \%)$ & UN \\
\hline Harmon et al. (2016) [26] & 45 & $12 / 45(27 \%)$ & $38(84 \%)$ & $\begin{array}{l}\text { LVEF } \leq 35 \% \text { or pacing indication } \\
\text { and LV GLS } \geq-15 \% \text { and/or NSVT/ } \\
\text { frequent PVBs with syncope or } \\
\text { planned HTX }\end{array}$ & $12(27 \%)$ & $27 / 45(60 \%)$ \\
\hline Chuzi et al. (2018) & 31 & $14 / 31(45 \%)$ & $25(80 \%)$ & Not specified & $2(6 \%)$ & $19 / 31(61 \%)$ \\
\hline Rezc et al. (2018) & 15 & $15 / 15(100 \%)$ & $14(93 \%)$ & NSVT and syncope/presyncope & $4(27 \%)$ & $13 / 15(87 \%)$ \\
\hline Kim et al. (2019) [62] & 23 & $7 / 23(30 \%)$ & $23(100 \%)$ & LVEF $\leq 35 \%$ or NSVT and/or syncope & $6(26 \%)$ & $14 / 23(61 \%)$ \\
\hline $\begin{array}{l}\text { Donellan et al. (2019) } \\
\text { [60] }\end{array}$ & 38 & $0 / 38(0 \%)$ & $35(92 \%)$ & Not specified & $8(21 \%)$ & UN \\
\hline
\end{tabular}

AL: Immunoglobulin light chain; CA: cardiac amyloidosis; HTX: heart transplantation; ICD: implantable cardioverter-defibrillator; LVEF: left ventricular ejection fraction; LV GLS: left ventricular global longitudinal strain; NSVT: nonsustained ventricular tachycardia; PVBs: premature ventricular beats; UN: unknown. 
selection and, importantly, is there a mortality benefit for ICD placement?

\section{Conclusion}

CA carries a high risk of SCD. However, ICD implantation for primary prevention of SCD remains controversial, and it is not clear whether ICDs improve survival in CA. Few data are available on arrhythmic SCD risk stratification, and the usual approach in these patients is secondary prevention or extrapolation of risk factors due to other cardiomyopathies, for example, impaired LV systolic dysfunction or NSVT, with or without syncope. However, their overall mortality is still high, particularly in patients in advanced stages of the disease, and they are, thus, at increased risk of death due to electromechanical dissociation. Therefore, the challenge is to identify at-risk patients in the early stage of the disease when $\mathrm{VA}$ risk predominates and to identify who may benefit from ICD therapy, mainly in the AL subtype. Accurate identification of patients who are more likely to die of an arrhythmia and less likely to die of other causes is required to ensure improvement in outcomes and wise use of resources. Incorporating methods that identify patients at particularly high risks of death from competing causes, who are unlikely to benefit from ICD therapy, will form an important part of this process. Further prospective studies are needed to understand the pathophysiology of cardiac arrhythmias in patients with CA, including electrophysiological study with endocardial mapping and modern CMR imaging, to indicate predictors of arrhythmic SCD and, finally, define the role of ICD in this group of patients.

\section{Author contributions}

Conception and design of the research; Acquisition of data; Analysis and interpretation of the data; Writing of the manuscript; Critical revision of the manuscript for intellectual content: Dumont CA, Sosa Liprandi MI.

\section{Potential Conflict of Interest}

No potential conflict of interest relevant to this article was reported.

\section{Sources of Funding}

There were no external funding sources for this study.

\section{Study Association}

This study is not associated with any thesis or dissertation work.

\section{Ethics approval and consent to participate}

This article does not contain any studies with human participants or animals performed by any of the authors.

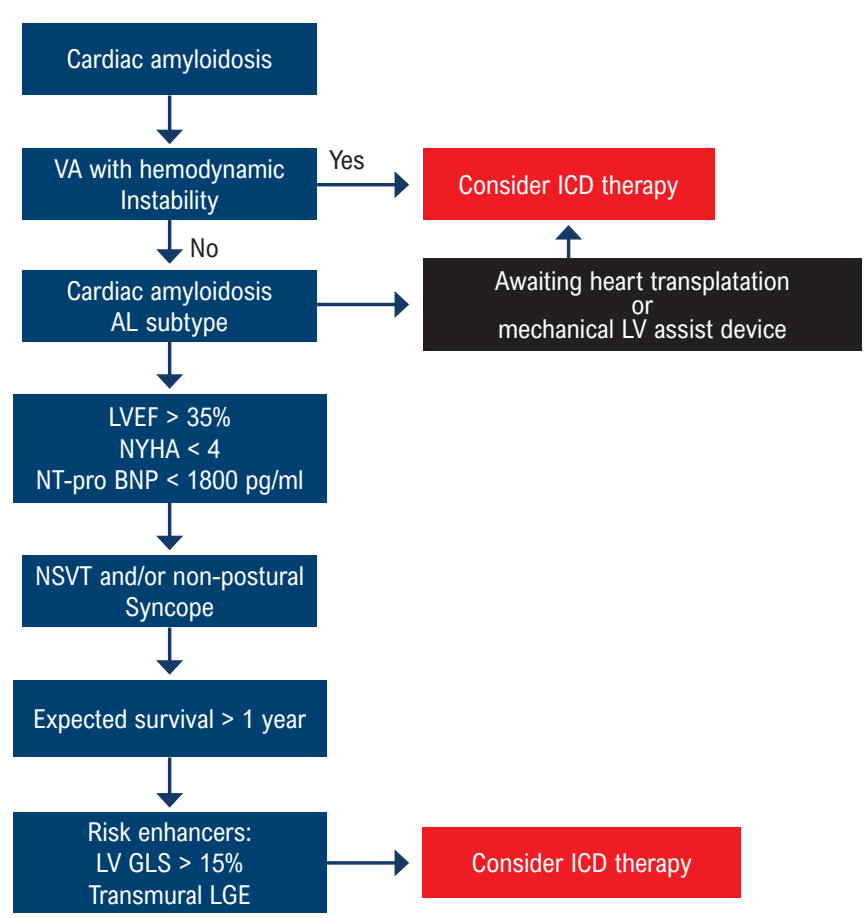

Figure 1 - Proposed algorithm for qualifying patients with cardiac amyloidosis for ICD implantation. AL: immunoglobulin light chain; ICD: implantable cardioverter-defibrillator; LGE: late gadolinium enhancement in cardiac magnetic resonance imaging; LV: left ventricular; LVEF: left ventricular ejection fraction; LV GLS: left ventricular global longitudinal strain assessed by speckle-tracking echocardiography; NT-proBNP: N-terminal B-type natriuretic peptide; NYHA: New York Heart Association; NSVT: nonsustained ventricular tachycardia; VA: ventricular arrhythmia. 


\section{References}

1. Falk RH. Diagnosis and Management of the Cardiac Amyloidoses. Circulation. 2005;112(13):2047-60. doi: 10.1161/CIRCULATIONAHA.104.489187.

2. Dubrey SW, Hawkins PN, Falk RH. Amyloid Diseases of the Heart: Assessment, Diagnosis, and Referral. Heart. 2011;97(1):75-84. doi: 10.1136/hrt.2009.190405.

3. Martinez-Naharro A, Hawkins PN, Fontana M. Cardiac Amyloidosis. Clin Med (Lond). 2018;18(Suppl 2):30-5. doi: 10.7861/clinmedicine.18-2-s30.

4. Lane T, Fontana M, Martinez-Naharro A, Quarta CC, Whelan CJ, Petrie A, et al. Natural History, Quality of Life, and Outcome in Cardiac Transthyretin Amyloidosis. Circulation. 2019;140(1):16-26. doi: 10.1161/ CIRCULATIONAHA.118.038169.

5. Escher F, Senoner M, Doerler J, Zaruba MM, Messner M, Mussner-Seeber C, et al. When and How do Patients with Cardiac Amyloidosis Die? Clin Res Cardiol. 2020;109(1):78-88. doi: 10.1007/s00392-019-01490-2.

6. Reisinger J, Dubrey SW, Lavalley M, Skinner M, Falk RH. Electrophysiologic Abnormalities in AL (Primary) Amyloidosis with Cardiac Involvement. J Am Coll Cardiol. 1997;30(4):1046-51. doi: 10.1016/s0735-1097(97)00267-2.

7. D’Errico S, Mazzanti A, Baldari B, Maiese A, Frati P, Fineschi V. Sudden Death in Lambda Light Chain AL Cardiac Amyloidosis: A Review of Literature and Update for Clinicians and Pathologists. Int J Clin Exp Pathol. 2020;13(7):1474-82.

8. Cappelli F, Vignini E, Martone R, Perlini S, Mussinelli R, Sabena A, et al. Baseline ECG Features and Arrhythmic Profile in Transthyretin Versus Light Chain Cardiac Amyloidosis. Circ Heart Fail. 2020;13(3):e006619. doi: 10.1161/CIRCHEARTFAILURE.119.006619.

9. Rochlani YM, Nishi SN, Hakeem A, Bhatti S. Burden of Arrhythmias in Patients Hospitalized with CardiacAmyloidosis. Circulation. 2015;132(Suppl3):A20097.

10. Sanchis K, Cariou E, Colombat M, Ribes D, Huart A, Cintas P, et al. Atrial Fibrillation and Subtype of Atrial Fibrillation in Cardiac Amyloidosis: Clinical and Echocardiographic Features, Impact on Mortality. Amyloid. 2019;26(3):128-38. doi: 10.1080/13506129.2019.1620724.

11. Palladini G, Malamani G, Cò F, Pistorio A, Recusani F, Anesi E, et al. Holter Monitoring in AL Amyloidosis: Prognostic Implications. Pacing Clin Electrophysiol. 2001;24(8):1228-33. doi: 10.1046/j.1460-9592.2001.01228.x.

12. Sayed RH, Rogers D, Khan F, Wechalekar AD, Lachmann HJ, Fontana M, et al. A Study of Implanted Cardiac Rhythm Recorders in Advanced Cardiac AL Amyloidosis. Eur Heart J. 2015;36(18):1098-105. doi: 10.1093/ eurheartj/ehu506.

13. Murtagh B, Hammill SC, Gertz MA, Kyle RA, Tajik AJ, Grogan M. Electrocardiographic Findings in Primary Systemic Amyloidosis and Biopsy-Proven Cardiac Involvement. Am J Cardiol. 2005;95(4):535-7. doi: 10.1016/j.amjcard.2004.10.028.

14. Goldsmith YB, Liu J, Chou J, Hoffman J, Comenzo RL, Steingart RM. Frequencies and Types of Arrhythmias in Patients with Systemic LightChain Amyloidosis with Cardiac Involvement Undergoing Stem Cell Transplantation on Telemetry monitoring. Am J Cardiol. 2009;104(7):990-4. doi: 10.1016/j.amjcard.2009.05.040.

15. Hörnsten R, Wiklund U, Olofsson BO, Jensen SM, Suhr OB. Liver Transplantation Does not Prevent the Development of Life-Threatening Arrhythmia in Familial Amyloidotic Polyneuropathy, Portuguese-Type (ATTR Val30Met) Patients. Transplantation. 2004;78(1):112-6. doi: 10.1097/01. tp.0000133517.20972.27.

16. Dubrey SW, Cha K, Anderson J, Chamarthi B, Reisinger J, Skinner M, Falk $\mathrm{RH}$. The Clinical Features of Immunoglobulin Light-Chain (AL) Amyloidosis with Heart Involvement. QJM. 1998;91(2):141-57. doi: 10.1093/ qjmed/91.2.141.

17. Ridolfi RL, Bulkley BH, Hutchins GM. The Conduction System in Cardiac Amyloidosis. Clinical and Pathologic Features of 23 Patients. Am J Med. 1977;62(5):677-86. doi: 10.1016/0002-9343(77)90870-1.
18. Li R, Yang ZG, Wen LY, Liu X, Xu HY, Zhang Q, et al. Regional Myocardial Microvascular Dysfunction in Cardiac Amyloid Light-Chain Amyloidosis: Assessment with 3T Cardiovascular Magnetic Resonance. J Cardiovasc Magn Reson. 2016;18:16. doi: 10.1186/s12968-016-0240-7.

19. Kumar S, Dispenzieri A, Lacy MQ, Hayman SR, Buadi FK, Colby C, et al. Revised Prognostic Staging System for Light Chain Amyloidosis Incorporating Cardiac Biomarkers and Serum Free Light Chain Measurements. J Clin Oncol. 2012;30(9):989-95. doi: 10.1200/ JCO.2011.38.5724.

20. Grogan M, Scott CG, Kyle RA, Zeldenrust SR, Gertz MA, Lin G, et al. Natural History of Wild-Type Transthyretin Cardiac Amyloidosis and Risk Stratification Using a Novel Staging System. J Am Coll Cardiol. 2016;68(10):1014-20. doi: 10.1016/j.jacc.2016.06.033.

21. Gillmore JD, Damy T, Fontana M, Hutchinson M, Lachmann HJ, MartinezNaharro A, et al. A New Staging System for Cardiac Transthyretin Amyloidosis. Eur Heart J. 2018;39(30):2799-806. doi: 10.1093/eurheartj/ehx589.

22. Varr BC, Zarafshar S, Coakley T, LiedtkeM, Lafayette RA, Arai S, etal. Implantable Cardioverter-Defibrillator Placement in Patients with Cardiac Amyloidosis. Heart Rhythm. 2014;11(1):158-62. doi: 10.1016/j.hrthm.2013.10.026.

23. Ashraf I, Peck MM, Maram R, Mohamed A, Crespo DO, Kaur G, et al. Association of Arrhythmias in Cardiac Amyloidosis and Cardiac Sarcoidosis. Cureus. 2020;12(8):e9842. doi: 10.7759/cureus.9842.

24. Pagourelias ED, Mirea O, Duchenne J, Van Cleemput J, Delforge M, Bogaert J, et al. Echo Parameters for Differential Diagnosis in Cardiac Amyloidosis: A Head-to-Head Comparison of Deformation and Nondeformation Parameters. Circ Cardiovasc Imaging. 2017;10(3):e005588. doi: 10.1161/ CIRCIMAGING.116.005588.

25. Falk RH, Rubinow A, Cohen AS. Cardiac Arrhythmias in Systemic Amyloidosis: Correlation with Echocardiographic Abnormalities. J Am Coll Cardiol. 1984;3(1):107-13. doi: 10.1016/s0735-1097(84)80436-2.

26. Hamon D, Algalarrondo V, Gandjbakhch E, Extramiana F, Marijon E, Elbaz N, et al. Outcome and Incidence of Appropriate Implantable Cardioverter-Defibrillator Therapy in Patients with Cardiac Amyloidosis. Int J Cardiol. 2016;222:562-8. doi: 10.1016/j.ijcard.2016.07.254.

27. Fluechter S, Kuschyk J, Wolpert C, Doesch C, Veltmann C, Haghi D, et al. Extent of Late Gadolinium Enhancement Detected by Cardiovascular Magnetic Resonance Correlates with the Inducibility of Ventricular Tachyarrhythmia in Hypertrophic Cardiomyopathy. J Cardiovasc Magn Reson. 2010;12(1):30. doi: 10.1186/1532-429X-12-30.

28. Fontana M, Pica S, Reant P, Abdel-Gadir A, Treibel TA, Banypersad SM, et al. Prognostic Value of Late Gadolinium Enhancement Cardiovascular Magnetic Resonance in Cardiac Amyloidosis. Circulation. 2015;132(16):1570-9. doi: 10.1161/CIRCULATIONAHA.115.016567.

29. Kotecha T, Martinez-Naharro A, Treibel TA, Francis R, Nordin S, Abdel-Gadir A, et al. Myocardial Edema and Prognosis in Amyloidosis. J Am Coll Cardiol. 2018;71(25):2919-31. doi: 10.1016/j.jacc.2018.03.536.

30. John RM. Arrhythmias in Cardiac Amyloidosis. J Innov Card Rhythm Manag. 2018;9(3):3051-7. doi: 10.19102/icrm.2018.090301.

31. Sidana S, Tandon N, Brady PA, Grogan M, Gertz MA, Dispenzieri A, et al. Prognostic Significance of Holter Monitor Findings in Patients with Light Chain Amyloidosis. Mayo Clin Proc. 2019;94(3):455-64. doi: 10.1016/j. mayocp.2018.08.039.

32. Lin G, Dispenzieri A, Kyle R, Grogan M, Brady PA. Implantable Cardioverter Defibrillators in Patients with Cardiac Amyloidosis. J Cardiovasc Electrophysiol. 2013;24(7):793-8. doi: 10.1111/jce.12123.

33. Halawa A, Woldu HG, Kacey KG, Alpert MA. Effect of ICD Implantation on Cardiovascular Outcomes in Patients with Cardiac Amyloidosis: A Systematic Review and Meta-Anaylsis. J Cardiovasc Electrophysiol. 2020;31(7):174958. doi: 10.1111/jce.14541. 
34. Towbin JA, McKenna WJ, Abrams DJ, Ackerman MJ, Calkins $\mathrm{H}$, Darrieux FCC, et al. 2019 HRS Expert Consensus Statement on Evaluation, Risk Stratification, and Management of Arrhythmogenic Cardiomyopathy. Heart Rhythm. 2019;16(11):301-72. doi: 10.1016/j. hrthm.2019.05.007.

35. Orini $M$, Graham AJ, Martinez-Naharro A, Andrews CM, de Marvao A, Statton B, Cook SA, O'Regan DP, Hawkins PN, Rudy Y, Fontana M, Lambiase PD. Noninvasive Mapping of the Electrophysiological Substrate in Cardiac Amyloidosis and Its Relationship to Structural Abnormalities. J Am Heart Assoc. 2019;8(18):e012097. doi: 10.1161/JAHA.119.012097.

36. Ramírez J, Orini M, Mincholé A, Monasterio V, Cygankiewicz I, Luna AB, et al. T-Wave Morphology Restitution Predicts Sudden Cardiac Death in Patients with Chronic Heart Failure. J Am Heart Assoc. 2017;6(5):e005310. doi: 10.1161/JAHA.116.005310.

37. Tilz RR, Lenarczyk R, Scherr D, Haugaa KH, lliodromitis K, Pürerfellner H, et al. Management of Ventricular Tachycardia in the Ablation Era: Results of the European Heart Rhythm Association Survey. Europace. 2018;20(1):209-13. doi: 10.1093/europace/eux332.

38. Alkindi S, Almasoud A, Younes A, Elamm C, Benatti R, Oliveira G, et al. Increased Risk of Heart Block in Patients with Cardiac Amyloidosis on Amiodarone. J Card Fail. 2015;21(8):125. doi: https://doi.org/10.1016/j. cardfail.2015.06.359.

39. Le Bras F, Molinier-Frenkel V, Guellich A, Dupuis J, Belhadj K, Guendouz $S$, et al. Sequential Cyclophosphamide-Bortezomib-Dexamethasone Unmasks the Harmful Cardiac Effect of Dexamethasone in Primary LightChain Cardiac Amyloidosis. Eur J Cancer. 2017;76:183-7. doi: 10.1016/j. ejca.2017.02.004

40. Goldberg MA, Antin JH, Guinan EC, Rappeport JM. Cyclophosphamide Cardiotoxicity: An Analysis of Dosing as a Risk Factor. Blood. 1986;68(5):1114-8.

41. Xiao Y, Yin J, Wei J, Shang Z. Incidence and Risk of Cardiotoxicity Associated with Bortezomib in the Treatment of Cancer: A Systematic Review and Meta-Analysis. PLoS One. 2014;9(1):e87671. doi: 10.1371/journal. pone.0087671.

42. Tan NY, Mohsin Y, Hodge DO, Lacy MQ, Packer DL, Dispenzieri A, et al. Catheter Ablation for Atrial Arrhythmias in Patients with Cardiac Amyloidosis. J Cardiovasc Electrophysiol. 2016;27(10):1167-73. doi: 10.1111/jce.13046.

43. Donnellan E, Wazni O, Kanj M, Elshazly MB, Hussein A, Baranowski B, et al. Atrial Fibrillation Ablation in Patients with Transthyretin Cardiac Amyloidosis. Europace. 2020;22(2):259-64. doi: 10.1093/europace/euz314.

44. Chung FP, Lin YJ, Kuo L, Chen SA. Catheter Ablation of Ventricular Tachycardia/Fibrillation in a Patient with Right Ventricular Amyloidosis with Initial Manifestations Mimicking Arrhythmogenic Right Ventricular Dysplasia/Cardiomyopathy. Korean Circ J. 2017;47(2):282-5. doi: 10.4070/ kcj.2016.0328.

45. Mlcochova H, Saliba WI, Burkhardt DJ, Rodriguez RE, Cummings JE, Lakkireddy D, et al. Catheter Ablation of Ventricular Fibrillation Storm in Patients with Infiltrative Amyloidosis of the Heart. J Cardiovasc Electrophysiol. 2006;17(4):426-30. doi: 10.1111/j.1540-8167.2005.00321.x.

46. Muser D, Santangeli P, Pathak RK, Castro SA, Liang JJ, Magnani S, et al. LongTerm Outcomes of Catheter Ablation of Ventricular Tachycardia in Patients with Cardiac Sarcoidosis. Circ Arrhythm Electrophysiol. 2016;9(8):e004333. doi: 10.1161/CIRCEP.116.004333.

47. Shumway SJ, Johnson EM, Svendsen CA, Kriett JM, Ring WS. Surgical Management of Ventricular Tachycardia. Ann Thorac Surg. 1997;63(6):1589-91. doi: 10.1016/s0003-4975(97)00358-5.

48. Estep JD, Bhimaraj A, Cordero-Reyes AM, Bruckner B, Loebe M, TorreAmione G. Heart Transplantation and End-Stage Cardiac Amyloidosis:
A Review and Approach to Evaluation and Management. Methodist Debakey Cardiovasc J. 2012;8(3):8-16. doi: 10.14797/mdcj-8-3-8.

49. Donnelly J, Soltesz E, Tong MZ, Hall SA, Kale P, Estep JD, et al. Heart Transplantation in Cardiac Amyloidosis: A Ten Year Experience. J Heart Lung Transplant. 2019;38(4):389. doi: https://doi.org/10.1016/j. healun.2019.01.989.

50. Maurer MS, SchwartzJH, Gundapaneni B, Elliott PM, Merlini G, WaddingtonCruz M, et al. Tafamidis Treatment for Patients with Transthyretin Amyloid Cardiomyopathy. N Engl J Med. 2018;379(11):1007-16. doi: 10.1056/ NEJMoa1805689.

51. Castaño A, Helmke S, Alvarez J, Delisle S, Maurer MS. Diflunisal for ATTR Cardiac Amyloidosis. Congest Heart Fail. 2012;18(6):315-9. doi: 10.1111/j.1751-7133.2012.00303.x.

52. Khanna S, Lo P, Cho K, Subbiah R. Ventricular Arrhythmias in Cardiac Amyloidosis: A Review of Current Literature. Clin Med Insights Cardiol. 2020;14:1179546820963055. doi: 10.1177/1179546820963055.

53. Priori SG, Blomström-Lundqvist C, Mazzanti A, Blom N, Borggrefe M, Camm J, et al. 2015 ESC Guidelines for the Management of Patients with Ventricular Arrhythmias and the Prevention of Sudden Cardiac Death: The Task Force for the Management of Patients with Ventricular Arrhythmias and the Prevention of Sudden Cardiac Death of the European Society of Cardiology (ESC). Europace. 2015;17(11):1601-87. doi: 10.1093/europace/euv319.

54. Al-Khatib SM, Stevenson WG, Ackerman MJ, Bryant WJ, Callans DJ, Curtis AB, et al. 2017 AHA/ACC/HRS Guideline for Management of Patients with Ventricular Arrhythmias and the Prevention of Sudden Cardiac Death: Executive Summary: A Report of the American College of Cardiology/ American Heart Association Task Force on Clinical Practice Guidelines and the Heart Rhythm Society. Circulation. 2018;138(13):210-e71. doi: 10.1161/CIR.0000000000000548

55. Kristen AV, Dengler TJ, Hegenbart U, Schonland SO, Goldschmidt H, Sack FU, et al. Prophylactic Implantation of Cardioverter-Defibrillator in Patients with Severe Cardiac Amyloidosis and High Risk for Sudden Cardiac Death. Heart Rhythm. 2008;5(2):235-40. doi: 10.1016/j.hrthm.2007.10.016.

56. Cheung CC, Roston TM, Andrade JG, Bennett MT, Davis MK. Arrhythmias in Cardiac Amyloidosis: Challenges in Risk Stratification and Treatment. Can J Cardiol. 2020;36(3):416-23. doi: 10.1016/j.cjca.2019.11.039.

57. Bart NK, Thomas L, Korczyk D, Atherton IJ, Stewart G], Fatkin D. Amyloid Cardiomyopathy. Heart Lung Circ. 2020;29(4):575-83. doi: 10.1016/j. hlc.2019.11.019.

58. John RM, Stern DL. Use of Implantable Electronic Devices in Patients with Cardiac Amyloidosis. Can J Cardiol. 2020;36(3):408-15. doi: 10.1016/j. cjca.2019.12.002.

59. Boldrini M, Salinaro F, Mussinelli R, Raimondi A, Alogna A, Musca F, et al. Prevalence and Prognostic Value of Conduction Disturbances at the Time of Diagnosis of Cardiac AL Amyloidosis. Ann Noninvasive Electrocardiol. 2013;18(4):327-35. doi: 10.1111/anec.12032.

60. Donnellan E, Wazni OM, Saliba WI, Baranowski B, Hanna M, Martyn M, et al. Cardiac Devices in Patients with Transthyretin Amyloidosis: Impact on Functional Class, Left Ventricular Function, Mitral Regurgitation, and Mortality. J Cardiovasc Electrophysiol. 2019;30(11):2427-32. doi: 10.1111/ jce.14180.

61. Kittleson MM, Maurer MS, Ambardekar AV, Bullock-Palmer RP, Chang PP Eisen HJ, et al. Cardiac Amyloidosis: Evolving Diagnosis and Management: A Scientific Statement from the American Heart Association. Circulation. 2020;142(1):7-22. doi: 10.1161/CIR.0000000000000792.

62. Kim EJ, Holmes B, Huang S, Lugo R, Al Aboud A, Richardson T, et al. Does an Implantable Defibrillator Alter Outcomes in Patients with Cardiac Amyloidosis? J Am Coll Cardiol. 2019;73(9):837. doi: 10.1016/S07351097(19)31444-5. 


\section{Review Article}

